\title{
Autoantibodies Specific to ER $\alpha$ are Involved in Tamoxifen Resistance in Hormone Receptor Positive Breast Cancer
}

\author{
Angela Maselli ${ }^{1}$, Stefania Parlato ${ }^{2}$, Rossella Puglisi ${ }^{1}$, Carla Raggi $^{3}$, Massimo Spada ${ }^{2}$, \\ Daniele Macchia ${ }^{2}$, Giada Pontecorvi ${ }^{1}$, Elisabetta Iessi ${ }^{1}$, Maria Teresa Pagano ${ }^{1}$, \\ Francesca Cirulli ${ }^{4}$, Lucia Gabriele ${ }^{2}$, Alessandra Carè ${ }^{1}$ (D), Patrizia Vici ${ }^{5}$, Laura Pizzuti ${ }^{5}$, \\ Maddalena Barba ${ }^{5}$, Paola Matarrese ${ }^{1, \dagger}$, Marina Pierdominici ${ }^{1,+}$ and Elena Ortona ${ }^{1, *,+}$ \\ 1 Center for Gender Specific Medicine, Istituto Superiore di Sanità, 00161 Rome, Italy \\ 2 Department of Oncology and Molecular Medicine, Istituto Superiore di Sanità, 00161 Rome, Italy \\ 3 National Centre for the Control and the Evaluation of Medicines, Istituto Superiore di Sanità, 00161 Rome, Italy \\ 4 Center for Behavioral Sciences and Mental Health, Istituto Superiore di Sanità, 00161 Rome, Italy \\ 5 Division of Medical Oncology 2, IRCCS Regina Elena National Cancer Institute, 00128 Rome, Italy \\ * Correspondence: elena.ortona@iss.it; Tel.: +39-0649902573 \\ + To be considered as senior authors.
}

Received: 29 May 2019; Accepted: 16 July 2019; Published: 19 July 2019

\begin{abstract}
Tamoxifen resistance is a major hurdle in the treatment of estrogen receptor (ER)-positive breast cancer. The mechanisms of tamoxifen resistance are not fully understood although several underlying molecular events have been suggested. Recently, we identified autoantibodies reacting with membrane-associated ER $\alpha$ (anti-ER $\alpha \mathrm{Abs}$ ) in sera of breast cancer patients, able to promote tumor growth. Here, we investigated whether anti-ER $\alpha$ Abs purified from sera of ER-positive breast cancer patients could contribute to tamoxifen resistance. Anti-ER $\alpha$ Abs inhibited tamoxifen-mediated effects on cell cycle and proliferation in MCF-7 cells. Moreover, anti-ER $\alpha$ Abs hampered the tamoxifen-mediated reduction of tumor growth in SCID mice xenografted with breast tumor. Notably, simvastatin-mediated disaggregation of lipid rafts, where membrane-associated ER $\alpha$ is embedded, restored tamoxifen sensitivity, preventing anti-ER $\alpha$ Abs effects. In conclusion, detection of serum anti-ER $\alpha$ Abs may help predict tamoxifen resistance and concur to appropriately inform therapeutic decisions concerning hormone therapy in ER-positive breast cancer patients.
\end{abstract}

Keywords: autoantibodies; estrogen receptor; breast cancer; tamoxifen; lipid rafts; statins

\section{Introduction}

Estrogens are known to have a major role in the onset and progression of breast cancer and almost $70 \%$ of breast tumors express estrogen receptor $\alpha(E R \alpha)$ [1-3]. ER $\alpha$ functions as a ligand dependent transcription factor that directly binds to specific estrogen responsive elements, thus regulating the transcription of estrogen-sensitive genes [4]. ER $\alpha$ was also shown to be associated with the plasma membrane (membrane associated $\mathrm{ER} \alpha, \mathrm{mER} \alpha$ ), concentrated in caveolae, where it transduces membrane-initiated estrogen-dependent activation of the mitogen-activated protein (MAP) kinase signaling pathway $[5,6]$.

The selective ER modulator (SERM) tamoxifen (TAM), which binds to and neutralizes ER $\alpha$, is one of the treatments of choice for ER-positive breast cancer patients for all stages of the disease in both pre- and post-menopausal women [1,7]. However, a large proportion of ER-positive patients show intrinsic or acquired drug resistance and relapse during or after endocrine therapy $[8,9]$. Several molecular mechanisms underlying TAM resistance have been suggested, including ER $\alpha$ 
mutations, ER $\alpha$ activation by growth factors and changes in TAM absorption, as well as activation of alternative signaling pathways [10]. In this context, a role for membrane-associated ER (mER $\alpha)$ signaling in TAM resistance has been also suggested [11,12].

Recently, we identified the presence of autoantibodies reacting with $\operatorname{mER} \alpha$ (anti-ER $\alpha$ Abs) in sera of patients with breast cancer [13]. These anti-ER $\alpha$ Abs act as estrogen agonist triggering rapid extracellular signal-regulated kinase (ERK) phosphorylation, and inducing tumor cell proliferation. The capacity of anti-ER $\alpha$ Abs to stimulate MCF-7 cell growth suggests some potential implication in the resistance to endocrine treatments.

Hence, the aim of this study was to investigate if anti-ER $\alpha$ Abs, binding to and activating $m E R \alpha$, play a role in TAM resistance. To this purpose, we first investigated the ability of anti-ER $\alpha$ Abs to interfere with TAM effects in vitro on MCF-7 breast cancer cells, analyzing proliferation and cell cycle progression. We also performed in vivo studies on Severe Combined Immunodeficiency (SCID) mice orthotopically grafted with MCF-7 cells, analyzing tumor growth, proliferation and apoptosis. Then, we investigated whether the disaggregation of lipid rafts, where mER $\alpha$ is embedded, may affect the action of anti-ER $\alpha$ Abs and, accordingly, restore the sensitivity to TAM.

\section{Patients and Methods}

\subsection{Purification of Specific Autoantibodies from Patients' Sera}

Anti-ER $\alpha$ antibodies were purified from sera of 10 ER-positive breast cancer patients (median age: 54.5 years, range: $42-85$ ) at stage I-II prior to treatment administration, selected on the basis of their positivity for anti-ER $\alpha$ Abs from a cohort enrolled at the IRCCS Regina Elena National Cancer Institute of Rome. The study was reviewed and approved by the Local Ethical Committee at the Regina Elena National Cancer Institute (Prot. R.U. 948/15), and a written informed consent was obtained from all patients. All clinical investigations have been conducted according to the principles expressed in the Declaration of Helsinki. Anti-ER $\alpha$ Ab purification was performed as previously described [13]. Briefly, total immunoglobulins were first purified by Protein A Antibody Purification Kit (Sigma Aldrich, St. Louis, MO, USA). Then, anti-ER $\alpha$ Abs were purified by affinity with the recombinant ER $\alpha$ spotted onto a nitrocellulose filter. After immunoglobulin incubation, specific anti-ER $\alpha$ Abs were eluted with $100 \mathrm{mM}$ glycine, $\mathrm{pH}$ 2.5, immediately neutralized with $1 \mathrm{M}$ Tris $\mathrm{HCl}, \mathrm{pH} 8$, and dialyzed against PBS. $\mathrm{Ab}$ preparation was analyzed by SDS-PAGE (4-12\% Invitrogen precast gel) and protein bands were visualized by Coomassie brilliant blue staining (Supplementary Figure S1). Endotoxin contamination of antibodies was determined by the quantitative chromogenic Limulus amebocyte cell lysate assay (QCL-1000; BioWhittaker, Walkersville, MD, USA). Antibodies from a preparation of intravenous immunoglobulin (IVIG) precipitated by saturated ammonium sulfate solution were used as control.

\subsection{Cell Culture and Treatment}

MCF-7 (ATCC HTB-22TM) cell line was obtained from the American Type Culture Collection (ATCC). Cells were cultured in DMEM low glucose $(1 \mathrm{~g} / \mathrm{L})$ without phenol red (Gibco BRL, Grand Island, NY, USA) supplemented with 10\% charcoal stripped fetal bovine serum (Euro-Clone, Pero, Milan, Italy), $2 \mathrm{mM}$ glutamine (Sigma Aldrich, St. Louis, MO, USA), and $50 \mathrm{mg} / \mathrm{mL}$ gentamycin (Sigma Aldrich, St. Louis, MO, USA) at $37^{\circ} \mathrm{C}$ in a humidified $5 \% \mathrm{CO}_{2}$ atmosphere and passaged for fewer than 6 months after receipt. On the basis of dose and time response curve constructed by incubating MCF-7 cells with serial dilutions of anti-ERa Abs (10-100 $\mu \mathrm{g} / \mathrm{mL})$ for 12, 24, 48 and $72 \mathrm{~h}$, we selected $50 \mu \mathrm{g} / \mathrm{mL}$ as the optimal concentration and $24 \mathrm{~h}$ as treatment time for in vitro experiments. Thus, cells were treated for $24 \mathrm{~h}$ with $50 \mu \mathrm{g} / \mathrm{mL}$ of human anti-ER $\alpha$ Abs or IVIG in presence or absence of TAM (Sigma Aldrich, St. Louis, MO, USA) $1 \mu \mathrm{M}$. In selected experiments cells were treated with 17- $\beta$ estradiol (E2) (Sigma Aldrich, St. Louis, MO, USA) $10 \mathrm{nM}$ or pre-treated with methyl-beta-cyclodextrin $(\mathrm{m} \beta C D)$ (Sigma-Aldrich, St. Louis, MO, USA) $2 \mathrm{mM}$ for $20 \mathrm{~min}$, or statin simvastatin (Sigma Aldrich, St. Louis, MO, USA) $2.5 \mu \mathrm{M}$ overnight before human anti-ER $\alpha$ Abs or IVIG addition. 


\subsection{Flow Cytometry}

\subsubsection{Apoptosis, Proliferation and Cell Cycle}

Apoptosis was quantified using a fluorescein isothiocyanate (FITC)-conjugated annexin V (AV) and propidium iodide (PI) detection kit according to the manufacturer's protocol (Marine Biological Laboratory, Woods Hole, MA, USA). This assay enables identification of both early (AV positive/PI negative) and late apoptotic or necrotic (PI positive) cells. Proliferation was evaluated by measuring Ki-67 nuclear antigen expression using the phycoerythrin (PE)-mouse anti-human Ki-67 set according to the manufacturer's protocol (BD Biosciences, San Jose, CA, USA). To analyze cell cycle progression, cells were synchronized at G1/S boundary by treatment with $0.7 \mu \mathrm{g} / \mathrm{mL}$ aphidicolin (Sigma), a specific DNA polymerase inhibitor, for $18 \mathrm{~h}$. After this time, cells were washed and then treated as stated above, or left untreated. Cells were then analyzed for cell cycle by the 5-bromo-2-deoxy-uridine (BrdU)/anti-BrdU method. Briefly, cells were pulse-labeled for 45 min with $30 \mu \mathrm{M}$ of BrdU (Sigma Aldrich, St. Louis, MO, USA). After this time, cells were processed as previously reported, resuspended in PBS containing 7-Aminoactinomycin D (7-AAD, Sigma Aldrich, St. Louis, MO, USA) and then analyzed for cell cycle by a biparametric flow cytometry analysis [14]. Samples were analyzed by collecting FL2 red fluorescence in a linear scale at $620 \mathrm{~nm}$ and FL1 green fluorescence in logarithmic scale at $512 \mathrm{~nm}$. Acquisition was performed on a dual-laser FACSCalibur flow cytometer (BD Biosciences, San Jose, CA, USA) and at least 30,000 events per sample were run. Data were analyzed using the Cell Quest Pro software (BD Biosciences, San Jose, CA, USA).

\subsubsection{Quantitative Fluorescence Resonance Energy Transfer (FRET)}

We applied FRET analysis by flow cytometry in order to quantify the localization of ER $\alpha$ within lipid rafts. Cells were stained with biotinylated cholera toxin B-subunit (CTxB), Invitrogen Corporation), used as a marker for lipid rafts, and with a specific mouse anti-ER $\alpha \mathrm{mAb}$ (C-311, Santa Cruz Biotechnology, Santa Cruz, CA, USA) for $1 \mathrm{~h}$ at $37^{\circ} \mathrm{C}$. After washings cells were incubated for $45 \mathrm{~min}$ at $37^{\circ} \mathrm{C}$ with $\mathrm{Cy} 5$-conjugated streptavidin (acceptor) and with an anti-mouse antibody conjugated with PE (donor) (both Sigma). After staining, cells were washed twice, resuspended in PBS and analyzed with a dual-laser FACSCalibur flow cytometer (BD Biosciences). For determination of FRET efficiency (FE), changes in fluorescence intensities of donor plus acceptor labeled cells were compared to the emission signal from cells labeled with donor-only and acceptor-only fluorophores. As a further control, the cross-reactivity among all the different primary and secondary antibodies was also assessed.

Efficient energy transfer resulted in an increased acceptor emission on cells stained with both donor and acceptor dyes. The FE was calculated according to Riemann [15] by using the following algorithm: $\mathrm{FE}=[\mathrm{FL} 3 \mathrm{DA}-\mathrm{FL} 2 \mathrm{DA} / \mathrm{a}-\mathrm{FL} 4 \mathrm{DA} / \mathrm{b}] /$ FL3DA, in which $\mathrm{A}$ is the acceptor and $\mathrm{D}$ the donor and where $\mathrm{a}=$ FL2D/FL3D and $\mathrm{b}=$ FL4A/FL3A. Fluorescence emission in channels two (PE), three (FRET) and four (Cy5) was expressed as median fluorescence.

\subsection{Fluorescence Microscopy}

For triple fluorescence microscopy analysis, living cells were stained as follows. For lipid rafts detection we used FITC-conjugated cholera toxin B (Sigma, St Louis, MO, USA); for ER $\alpha$ detection a specific mouse anti-ER $\alpha$ mAb (C-311, Santa Cruz Biotechnology, Santa Cruz, CA, USA) was used followed by the AlexaFluor 594-conjugated anti-mouse IgG (Invitrogen Corporation). After washing, all samples were counterstained with Hoechst 33258 (Sigma, $1 \mathrm{mg} / \mathrm{mL}$ in PBS) and then mounted in glycerol/PBS (ratio 1:1, pH 7.4). The images were acquired by intensified video microscopy (IVM) with an Olympus fluorescence microscope (Olympus Corporation of the Americas, Center Valley, PA, USA), equipped with a Zeiss charge-coupled device (CCD) camera (Carl Zeiss, Oberkochen, Germany). 


\subsection{Western Blot}

Breast tumor tissues were mechanically disrupted and lysed in RIPA buffer (150 mM sodium chloride, $1.0 \%$ Triton $\mathrm{X}-100,0.5 \%$ sodium deoxycholate, $0.1 \%$ SDS, $50 \mathrm{mM}$ Tris, $\mathrm{pH} 8.0$ ) plus protease inhibitors. $30 \mu \mathrm{g}$ total tumor lysates were separated on $12 \%$ acrylamide gels. After electrophoresis, the proteins resolved on the gel were electrophoretically transferred to a polyvinylidene difluoride (PVDF) membrane (Bio-Rad, Hercules, Richmond, CA, USA) and blocked for $1 \mathrm{~h}$ with $5 \%$ dry milk in TBS- $0.1 \%$ Tween. The membrane was probed with a primary rabbit antibody to Cyclin E (Upstate, Millipore, Darmstadt, Germany). After probing with a horseradish peroxidase (HRP)-conjugated anti-rabbit antibody (Jackson ImmunoResearch Laboratories, Baltimore Pike, West Grove, PA, USA), specific staining was visualized by enhanced chemiluminescence (ECL) Western detection system (Millipore, Darmstadt, Germany). To ensure the presence of equal amounts of proteins, the membranes were reprobed with rabbit antihuman HSC-70 (Santa Cruz Technology, Inc., Dallas, TX, USA). Quantification of protein expression was performed by Image J program (Bio-Rad, Richmond, CA, USA).

\subsection{Activity of Anti-ER $\alpha$ Abs in Tumor-Bearing Severe Combined Immunodeficient (SCID) Mice}

Female SCID mice were ovariectomized at 8 weeks of age and implanted subcutaneously with an E2 pellet ( $0.36 \mathrm{mg} 60$-day release/pellet). After ten days mice were implanted with $5 \times 10^{6} \mathrm{MCF}-7$ cells into the right mammary fat pad. In the different sets of experiments, at the onset of tumor (i.e., 10 days from MCF-7 cell injection), mice were randomized into the following treatment groups: (i) IVIG (10 $\mu \mathrm{g} /$ day); (ii) anti-ER $\alpha$ Abs (10 $\mu \mathrm{g} /$ day); (iii) TAM (Sigma Aldrich, St. Louis, MO, USA;

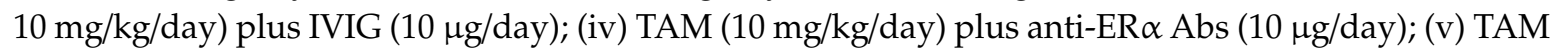
(10 mg/kg/day) plus IVIG (10 $\mathrm{\mu g} /$ day) plus simvastatin $(5 \mathrm{mg} / \mathrm{kg} /$ day); and (vi) TAM (10 mg/kg/day) plus anti-ER $\alpha$ Abs $(10 \mu \mathrm{g} /$ day) plus simvastatin $(5 \mathrm{mg} / \mathrm{kg} /$ day $)$. All treatments were administered 5 days/week, for 2 weeks, intraperitoneally. The tumor volumes were calculated using the following formula: $(\mathrm{a} \times \mathrm{b} 2) / 2$, where $\mathrm{a}$ and $\mathrm{b}$ represent the longest and shortest diameters, respectively.

The study was reviewed and approved by the Local Ethical Committee at the Italian Ministry of Health (protocol. n.708/2016-PR)

\subsection{Immunofluorescence Analysis on Formalin-Fixed, Paraffin-Embedded (FFPE) Tumor Tissues}

Serial sections from mice tumor nodules embedded in paraffin were dewaxed and rehydrated.

For immunolocalization studies slides were subjected to heat-mediated antigenic retrieval $(10 \mathrm{mM}$ Sodium Citrate buffer $\mathrm{pH}$ 6.0) and subsequently permeabilizated ( $0.1 \%$ Triton X-100 for $10 \mathrm{~min})$ and saturated ( $3 \%$ BSA for at least $2 \mathrm{~h}$ ) at room temperature. After incubation overnight at $4{ }^{\circ} \mathrm{C}$ in a humidified chamber with the primary anti-human Ki-67 antibody (Clone MIB-1; M7240, DAKO Glostrup, Denmark), slides were incubated with Alexa Fluor 647 conjugated anti-mouse antibody (Molecular Probes, Eugene, OR, USA) for $45 \mathrm{~min}$ at room temperature. Negative controls were performed by omission of the primary antibody in each experiment. Finally, slides were mounted with SlowFade anti-fade reagent containing DAPI (Molecular Probes, Eugene, OR, USA) and analyzed by Olympus F1000 laser-scanning confocal microscopy (Olympus, Tokyo, Japan). Ki-67 was scored by the average method: manually counting the positive tumor cells in at least 10 microscope fields for each sample and calculating the average percentage of positive tumor cells.

\subsection{Data Analysis and Statistic}

Statistical analysis was performed with the statistical package Prism 6 (GraphPad Software, La Jolla, CA, USA). Results are presented as the mean \pm standard deviation (SD) of the values obtained in at least three independent experiments. Comparisons between two groups were performed by Student's $t$ test.

$p<0.05$ was considered to indicate a statistically significant difference. 


\section{Results and Discussion}

\subsection{The Anti-Proliferative Effects of Tamoxifen is Inhibited by Anti-ER $\alpha$ Abs}

Endocrine resistance remains the major concern and limit to the use of endocrine therapy. It is now clear that TAM resistance is not triggered exclusively by one single mechanism but involves several functional pathways [10]. We have previously observed that human anti-ER $\alpha$ Abs were able to act as $m E R \alpha$ agonists inducing in vitro in breast cancer MCF-7 cell line the following effects: (i) rapid activation of ERK; and (ii) increase of cell proliferation [13]. Here, we investigated whether anti-ER $\alpha$ Abs could also lead to a modification in the susceptibility to the SERM TAM in MCF-7 cells. For this purpose, we purified anti-ER $\alpha$ Abs from sera of patients with ER positive breast cancer as described in the Methods section. We treated MCF-7 cells with: (i) human antibodies from a preparation of intravenous immunoglobulin (IVIG) $(50 \mu \mathrm{g} / \mathrm{mL})$; (ii) anti-ER $\alpha$ Abs (50 $\mu \mathrm{g} / \mathrm{mL})$; (iii) TAM $1 \mu$ M; (iv) IVIG $(50 \mu \mathrm{g} / \mathrm{mL}$ plus TAM $1 \mu \mathrm{M}$; (v) anti-ER $\alpha$ Abs $(50 \mu \mathrm{g} / \mathrm{mL})$ plus TAM $1 \mu \mathrm{M}$.

As expected, anti-ER $\alpha$ Abs significantly increased MCF-7 cell proliferation $(p=0.03)$. Notably, anti-ER $\alpha$ Abs were also able to significantly reverse the anti-proliferative effect of TAM $(p=0.0089)$ (Figure 1a). Similarly, analyzing cell cycle progression, anti-ER $\alpha$ Abs were able to significantly reduce the G1 arrest induced by TAM treatment $(p=0.0148)$ (Figure 1b).
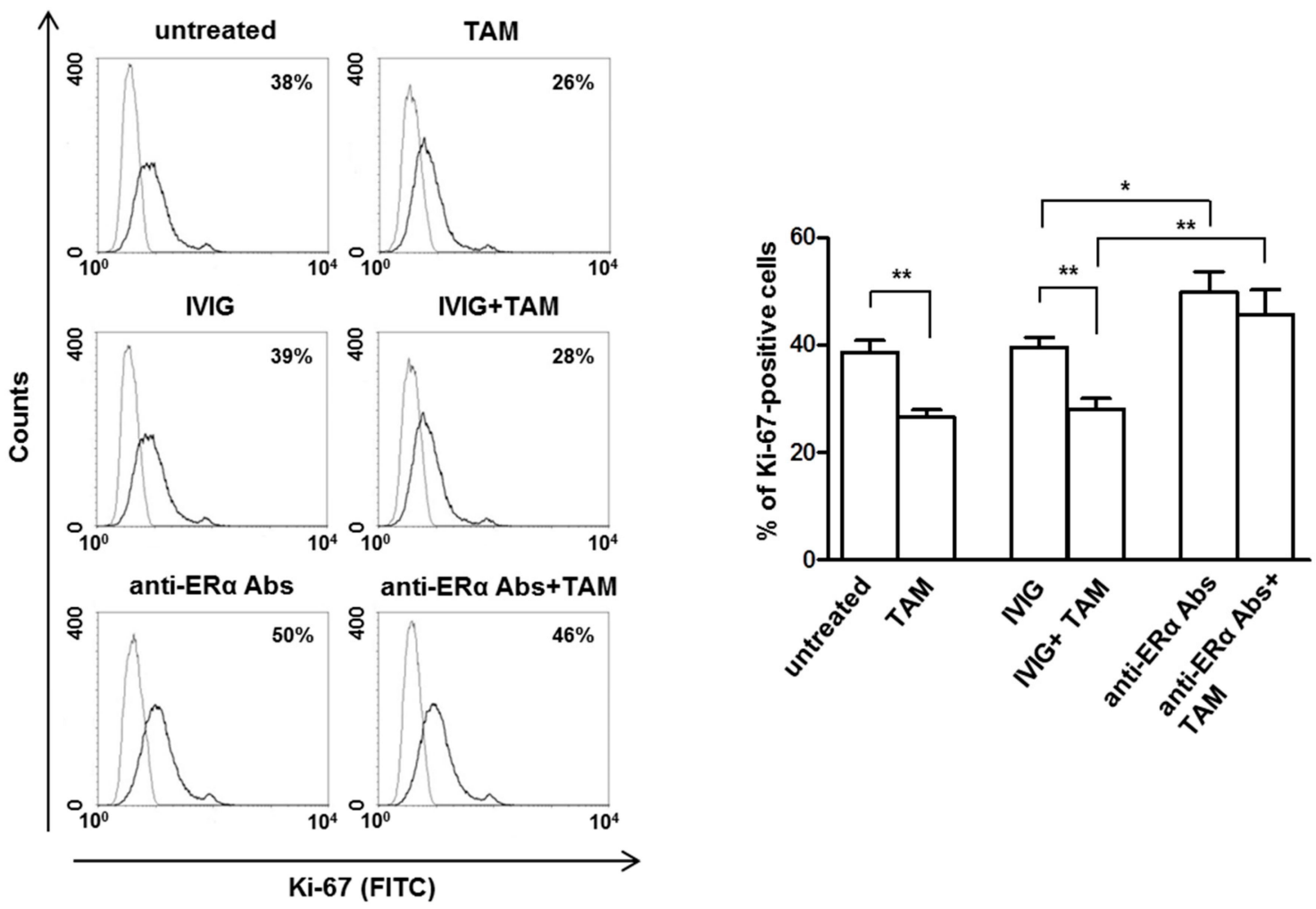

(a)

Figure 1. Cont. 

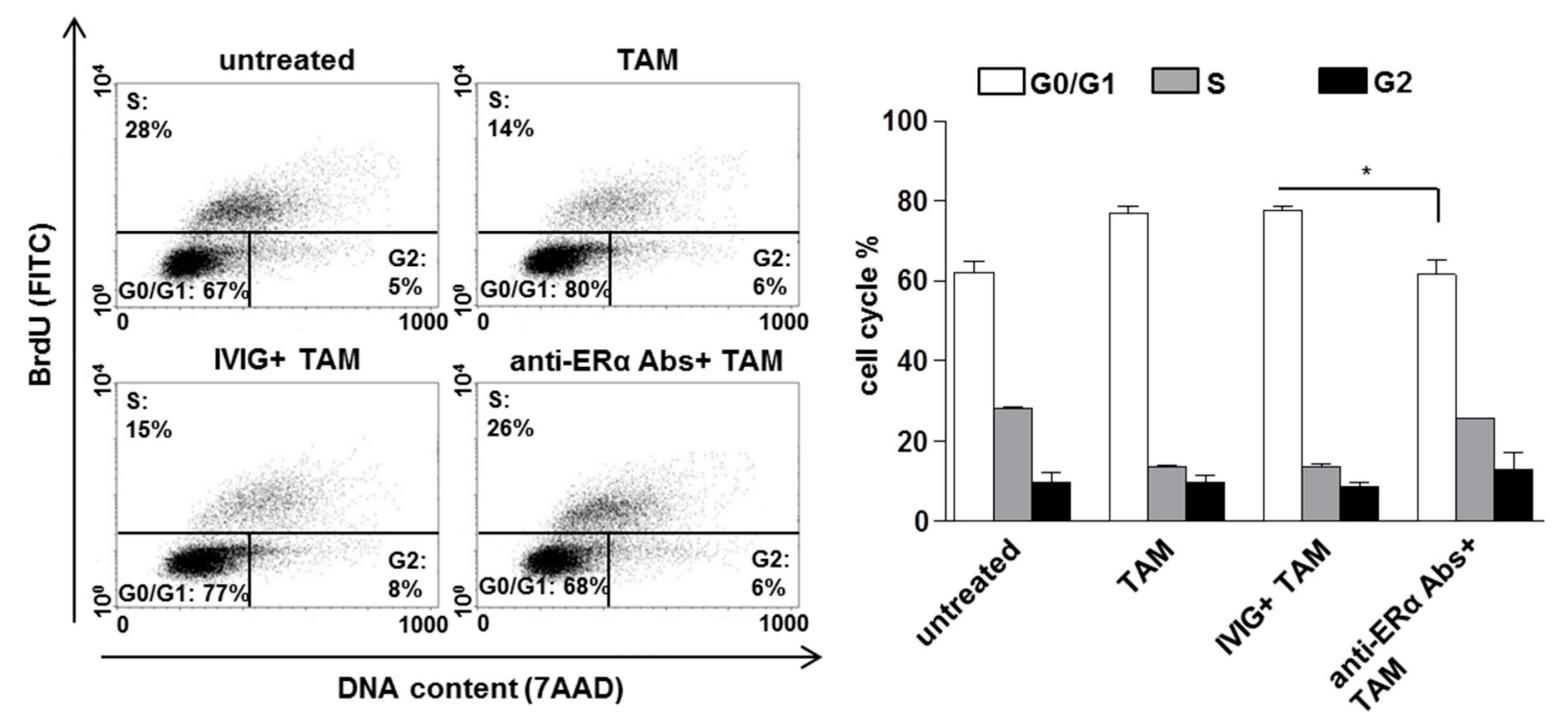

(b)

Figure 1. Anti-ER $\alpha$ Abs revert the effect of tamoxifen (TAM) on cell proliferation and cell cycle. (a) Cell proliferation was evaluated by flow cytometry measuring Ki-67 nuclear antigen expression in MCF-7 cells treated or not for $24 \mathrm{~h}$ with intravenous immunoglobulin (IVIG) (50 $\mu \mathrm{g} / \mathrm{mL})$, anti-ER $\alpha$ Abs $(50 \mu \mathrm{g} / \mathrm{mL})$, TAM $1 \mu \mathrm{M}$, IVIG $(50 \mu \mathrm{g} / \mathrm{mL})$ plus TAM $1 \mu \mathrm{M}$, anti-ER $\alpha$ Abs $(50 \mu \mathrm{g} / \mathrm{mL})$ plus TAM 1 $\mu \mathrm{M}$. Results from one representative experiment out of five are shown (left). Data are also reported as mean $\pm \mathrm{SD}$ (right), ${ }^{*}, p<0.05 ;{ }^{* *}, p<0.01$ by Student's $t$ test. (b) Cell-cycle phases were determined by flow cytometry analysis of BrdU/7AAD staining in synchronized MCF-7 cells treated or not for $24 \mathrm{~h}$ with TAM $1 \mu \mathrm{M}$, IVIG $(50 \mu \mathrm{g} / \mathrm{mL})$ plus TAM $1 \mu \mathrm{M}$, anti-ER $\alpha$ Abs $(50 \mu \mathrm{g} / \mathrm{mL})$ plus TAM $1 \mu \mathrm{M}$. Results from one representative experiment out of five are shown. Data are also reported as mean $\pm \mathrm{SD}$ of each phase. ${ }^{*}, p<0.05$ by Student's $t$ test.

We also tested the effect of 17- $\beta$ estradiol (E2) in combination with anti-ER $\alpha$ Abs on MCF-7 cell proliferation (Supplementary Figure S2) and we observed that: (i) E2 induced MCF-7 cell proliferation; (ii) anti-ER $\alpha$ Abs increased E2-mediated cell proliferation; (iii) TAM inhibited E2-mediated cell proliferation; (iv) anti-ER $\alpha$ Abs inhibited the TAM effect also in presence of E2. Our data suggest that strong membrane ER $\alpha$ activation by anti-ER $\alpha$ Abs could inhibit the antagonist properties of TAM, promoting endocrine resistance. This effect occurred in presence or absence of estrogen, conditions resembling pre- and post-menopausal status.

To further evaluate these effects in vivo, MCF-7 cells were transplanted into SCID mice and TAM alone or in association with anti-ER $\alpha$ Abs were administered at the onset of tumor.

Differently from what was observed in vitro, anti-ER $\alpha$ Abs did not significantly increase the in vivo growth of MCF-7 tumor (Figure 2a), despite an increase of proliferative cells rates (i.e., Ki-67-positive cells) was observed (IVIG treatment vs. anti-ER $\alpha$ Abs treatment, $p=0.01$, Figure $2 b$ ). Treatment with TAM plus IVIG reduced tumor growth, and, according to what observed in vitro, although less evident, this effect was significantly hampered by the presence of anti-ER $\alpha$ Abs $(p=0.0003$ at 14 th day after treatment, Figure 2a). In the same vein, the anti-proliferative effect of TAM was significantly reduced by the presence of anti-ER $\alpha$ Abs (TAM + IVIG vs. TAM + anti-ER $\alpha$ Abs, $p=0.03$, Figure 2c). 


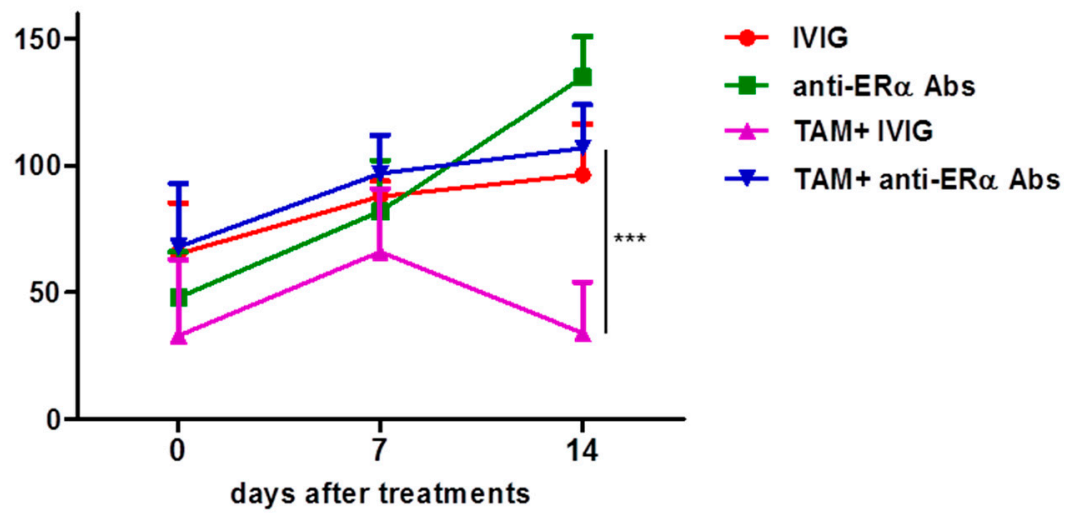

(a)

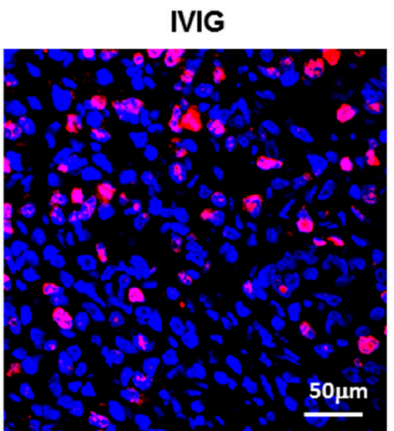

Im

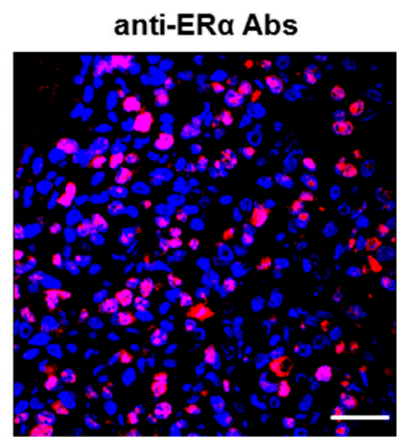

(b)

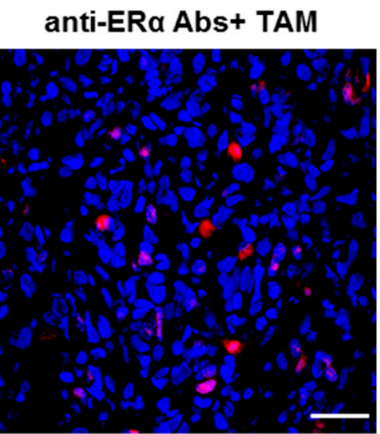

(c)
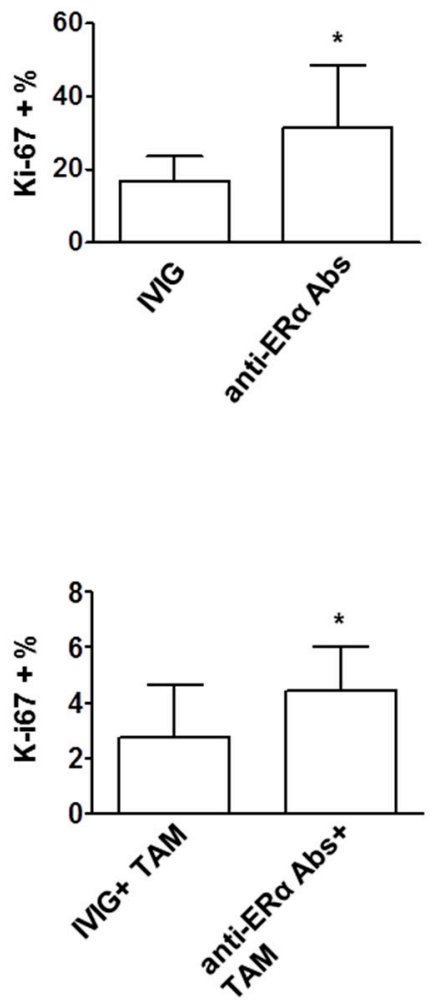

Figure 2. Anti-ER $\alpha$ Abs induced tumor growth and TAM resistance in breast cancer xenografts in Severe Combined Immunodeficiency (SCID) mice. (a) The mean $( \pm$ SD) of the tumor volume of one set of experiment (10 mice/group) was shown. The tumor volumes were calculated at day 0 , day 7 and day 14 using the following formula: $(\mathrm{a} \times \mathrm{b} 2) / 2$, where $\mathrm{a}$ and $\mathrm{b}$ represent the longest and shortest diameters, respectively. At day 14, TAM + IVIG vs. TAM + anti-ER $\alpha$ Abs: ${ }^{* * *}, p<0.001$ by Student's $t$ test. $(\mathbf{b}, \mathbf{c})$ Representative sections from MCF-7 tumors treated with IVIG, anti-ER $\alpha$ Abs, TAM + IVIG and TAM + anti-ER $\alpha$ Abs immunostained with anti-Ki67 (red) antibodies and counterstained with Hoechst (blue) are shown. Only nuclear staining (plus mitotic figures) was considered positive. Ki-67 was scored by the average method as reported in Methods. Results are represented as mean \pm SD. Statistical analysis: ${ }^{*}, p<0.05$ by Student's $t$ test. Scale bar $=50 \mu \mathrm{m}$.

Summarizing the results of this first set of experiments, anti-ER $\alpha$ Abs purified from sera of ER-positive breast cancer patients are able to inhibit the in vitro TAM-mediated effects on cell cycle 
and proliferation of MCF-7 cells. Moreover, anti-ER $\alpha$ Abs hampered the TAM-mediated reduction of tumor growth in SCID mice xenografted with breast tumor. These first time findings suggest a role for serum anti-ER $\alpha$ Abs in the occurrence of TAM resistance, being able to induce tumor proliferation and growth, overcoming TAM effect. These data suggest that the signaling mediated by anti-ER $\alpha$ Abs-membrane ER interferes with ER making it no longer responsive to TAM. Further studies are needed to clarify the molecular mechanism underlying this effect.

From a clinical point of view, the possible use of anti-ER $\alpha$ Abs as potential predictive biomarkers for resistance to TAM may be assumed and future longitudinal clinical studies are needed to verify this hypothesis in adequately sized, ad hoc trials. In addition, hitting anti-ER $\alpha \mathrm{Ab}$ target, i.e., $\mathrm{mER} \alpha$, may open new perspectives to overcome resistance to TAM, at least in patients positive for anti-ER $\alpha$ Abs. Hence, the second set of experiments of this study was carried out to evaluate the possibility of interfering with $\mathrm{mER} \alpha$ to restore TAM sensitivity.

\subsection{Localization of $m E R \alpha$ in Lipid Rafts}

The functions performed by anti-ER $\alpha$ Abs are initiated at the plasma membrane level, throughout the interaction with $\mathrm{mER}$, which is located within the lipid rafts [5], plasma membrane microstructures with a distinct lipid composition. Cholesterol, in addition to merely being a membrane component required for fluidity, is an integral component of lipid rafts and plays a role in subsequent membrane associated signaling events. Cholesterol-enriched lipid raft domains are highly expressed in tumor cells and are described as "survival pools" for promoting pro-survival and pro-proliferation pathways, both of which are targets for cancer prevention and therapy $[16,17]$. Thus, it is possible that excess cholesterol might increase signaling events thereby promoting breast cancer progression. Interestingly, TAM resistant cells in comparison with TAM responsive cells constitutively express higher levels of cholesterol-enriched lipid raft domains. Caveolae-related domains in breast cancer cells showed significant enrichment of human epidermal growth factor receptor (HER) and $m E R \alpha$ [18]. Thus, HER receptor and $\mathrm{mER} \alpha$ converge at the level of lipid rafts where they coexist in close physical proximity and may cooperate in stimulating cancerous growth and TAM resistance [18-20].

With this in mind, we analyzed the localization of $\mathrm{mER} \alpha$ in lipid rafts even in the presence of cholesterol modulators, such as methyl-beta-cyclodextrin $(\mathrm{m} \beta \mathrm{CD})$ and the statin simvastatin. As shown in Figure 3a, mER $\alpha$ was substantially localized within lipid rafts in MCF-7 cells (left picture, yellow fluorescence). After treatment with $\mathrm{m} \beta C D$, a compound that alters lipid microdomains by removing cholesterol from the membranes [21], localization of $\mathrm{mER} \alpha$ in lipid rafts, and their structural organization, were completely lost (Figure $3 a$, middle picture). Overlapping results were obtained by treating MCF-7 cells with the cholesterol-lowering drug simvastatin (Figure 3a, right picture). Then, we analyzed the localization of $m E R \alpha$ within lipid rafts by means of quantitative FRET technique using the $\mathrm{CTxB}$, able to bind with high avidity gangliosides, enriched in the plasma membrane lipid rafts, and a specific antibody to $E R \alpha$. We observed a significant decrease of $m E R \alpha$ localization within lipid rafts alike in cells treated with $\mathrm{m} \beta C D$ or simvastatin, as indicated by fluorescence resonance energy transfer (FRET) efficiency (FE) values calculated according to Riemann's algorithm (Figure 3b,c). 

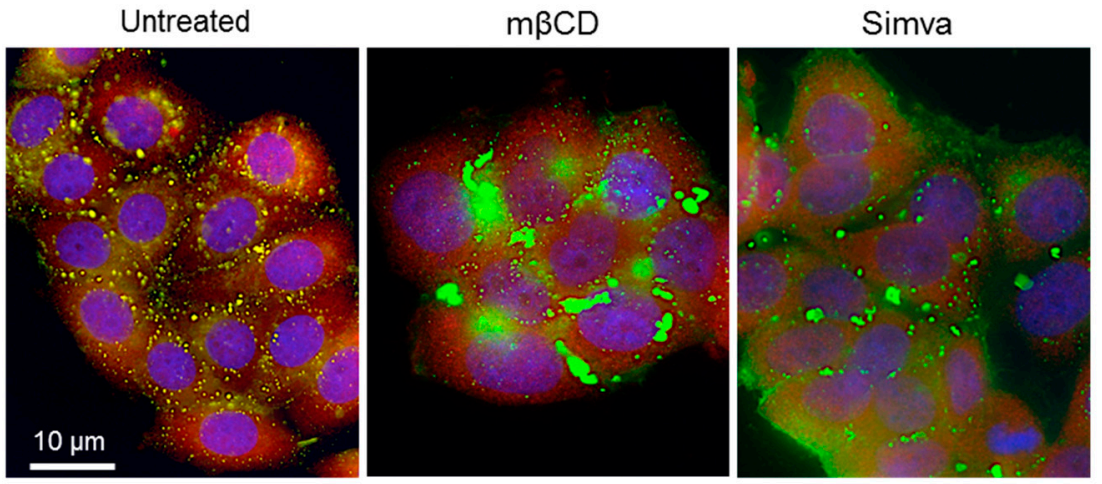

(a)
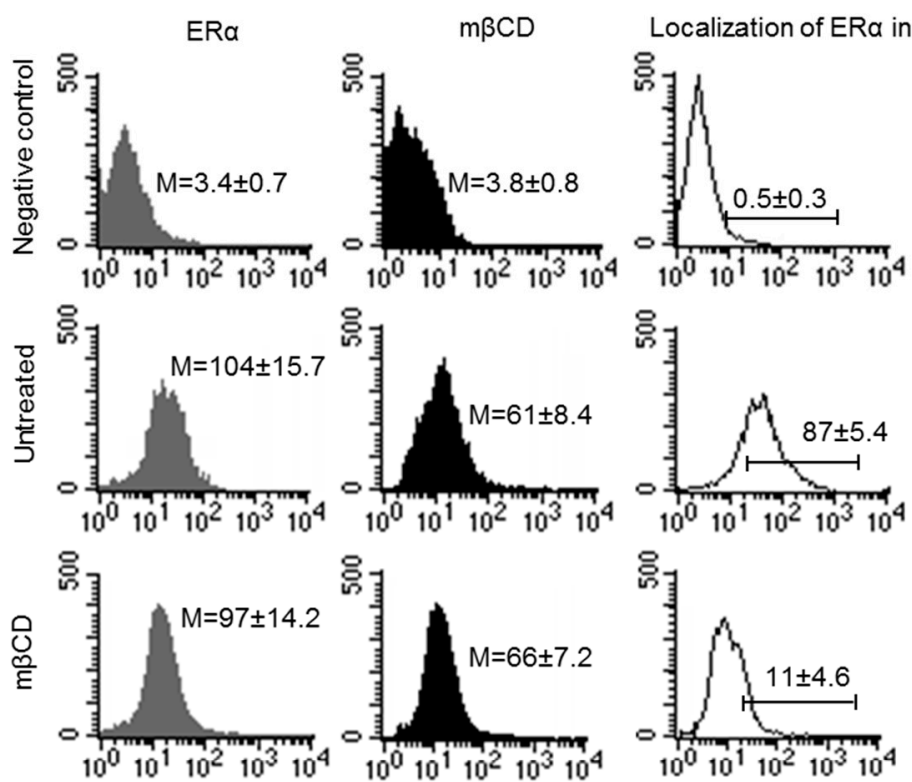

Untreated

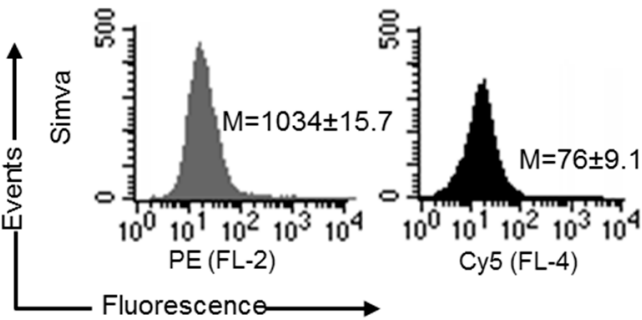

(b)

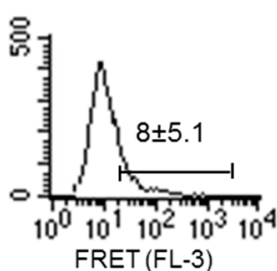

FRET (FL-3)

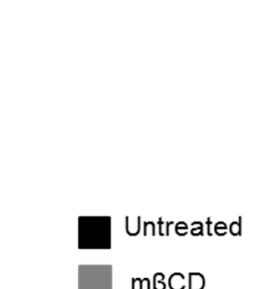

$m \beta C D$

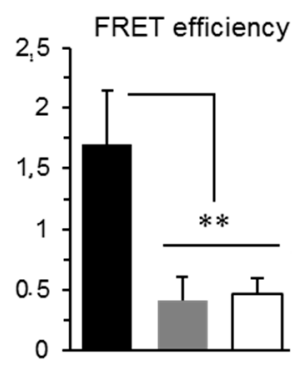

(c)

Figure 3. Localization of $m E R \alpha$ within lipid rafts. (a) intensified video microscopy (IVM) analysis after triple cell staining with fluorescein isothiocyanate (FITC)-conjugated CTxB (green)/ER $\alpha$ (red)/Hoechst (blue) in MCF-7 cells untreated or treated with $\mathrm{m} \beta \mathrm{CD}$ or simvastatin. Yellow fluorescence indicates localization of ER $\alpha$ within lipid rafts. Magnification, 700×. (b) Quantitative evaluations by fluorescence resonance energy transfer (FRET) technique of the localization of $\mathrm{mER} \alpha$ within lipid rafts (labeled by $\mathrm{CTxB}$ ), as revealed by flow cytometry analysis. Numbers in the first and second columns (grey and black histograms) indicate the median fluorescence intensity. In the third column (empty histograms) the percentage of FL3-positive events, obtained in one experiment representative of three, is shown. (c) Bar graph showing the FRET efficiency, calculated according to the Riemann's algorithm. Data are reported as mean \pm SD from three independent experiments. ${ }^{* *}, p<0.01$ vs. untreated cell samples by Student's $t$ test. Simva: simvastatin. 
Taken together, these data suggested that the localization of $\mathrm{mER} \alpha$ in lipid raft was completely lost altering lipid microdomains by removing or lowering cholesterol from membranes with $\mathrm{m} \beta C D$ or with simvastatin.

\subsection{Perturbation of Lipid Rafts is a Potential Approach to Restore TAM Effects in Presence of Anti-ER $\alpha$ Abs}

A clinical benefit of statins on reducing cancer recurrence and mortality, with limited effects on the incidence of primary cancer has been reported. A number of basic research studies have shown that statins can reduce tumor cell growth and proliferation by inducing cell cycle arrest [22].

Hence, on the basis of the results reported above, we evaluated if treatment with statins was able to restore the antitumor effect of TAM on MCF-7 cells challenged with human anti-ER $\alpha$ Abs. As shown in Figure $4 a, b$, simvastatin was able to significantly inhibit anti-ER $\alpha$ Abs effect on proliferation and cell cycle progression in MCF-7 cell treated with TAM ( $p=0.023$ and $p=0.0025$, respectively). Moreover, in SCID mice bearing MCF-7 tumor xenografts, the administration of simvastatin hampered the anti-ER $\alpha$ Abs mediated inhibition of TAM effect (i.e., reduction of tumor volume) $(p<0.0001$, Figure $4 c)$. Analyzing tumor nodules, we also observed that simvastatin was able to mitigate the anti-ER $\alpha$ Abs inhibitory effect on TAM action. In fact, in presence of simvastatin, no significant difference was observed in term of Ki-67 expression in tumor tissue independently on anti-ER $\alpha$ Abs (Figure $4 \mathrm{~d}$ ). To further support the ability of simvastatin to inhibit the effect of anti-ER $\alpha$ Abs on TAM, we also analyzed in tumor tissue the expression of cyclin E that drives the transition from G1 to $S$ phase, leading to the initiation of DNA synthesis [23,24]. According to in vitro experiments, simvastatin was able to prevent the significant increase of cyclin E level observed in the presence of anti-ER $\alpha$ Abs $(p=0.0036$, Figure 4e).

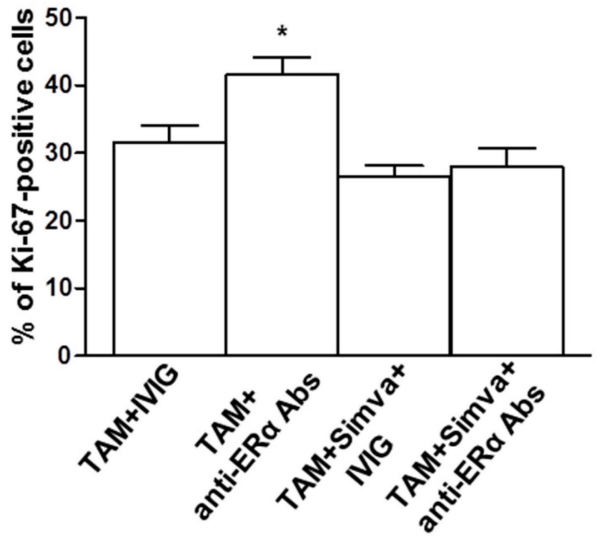

(a)

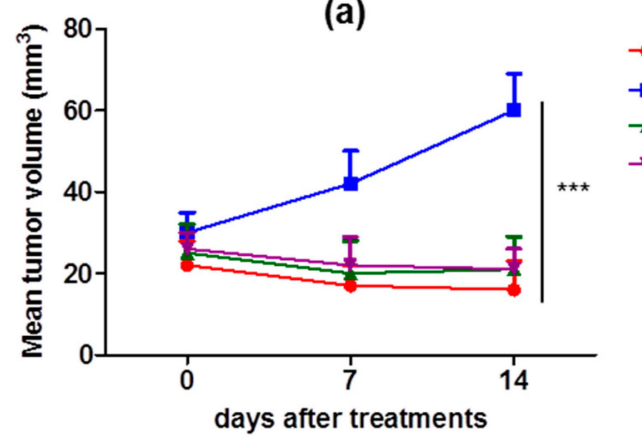

(c)

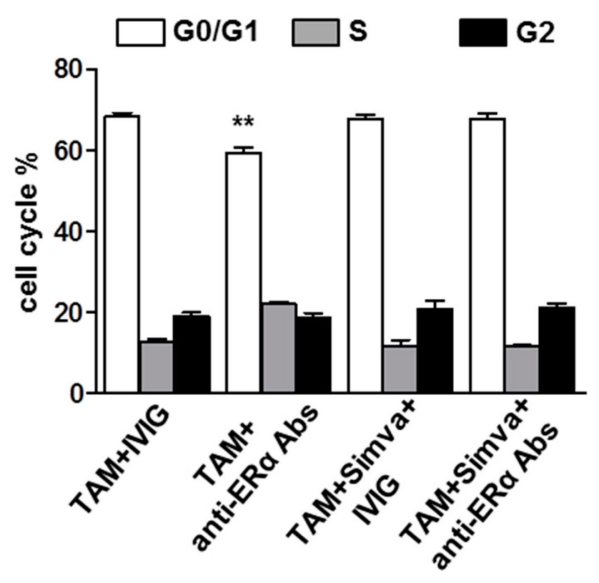

(b)

Figure 4. Cont. 

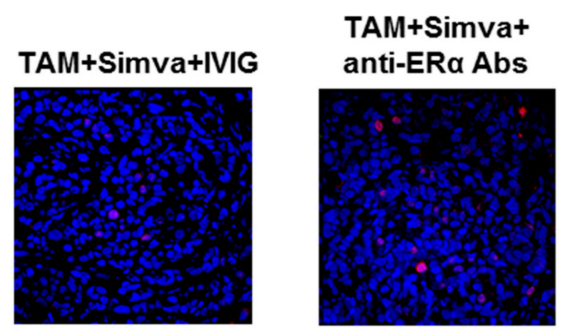

(d)
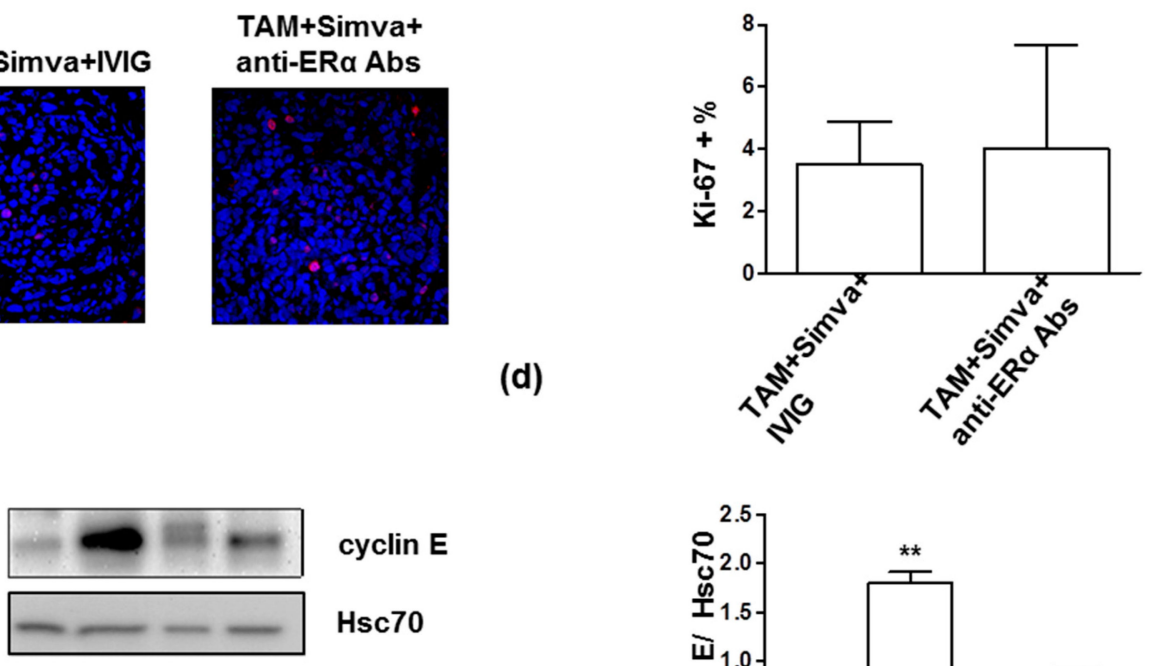

cyclin E

Hsc70

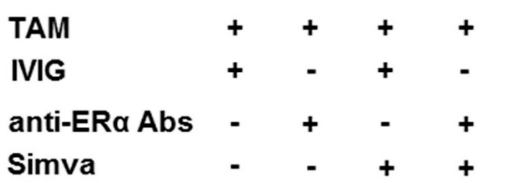

(e)

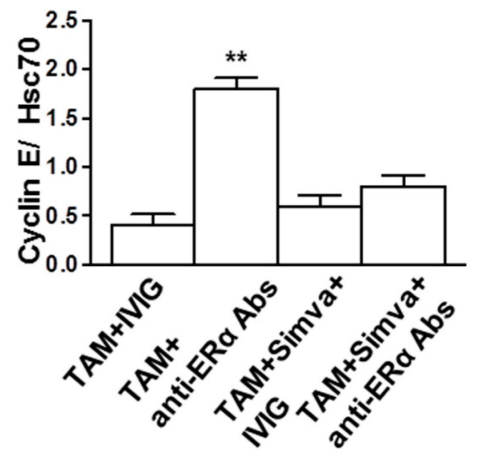

Figure 4. Simvastatin treatment, blocking anti-ER $\alpha$ Abs action, restored TAM effect both in vitro and in vivo. (a) Cell proliferation was evaluated by flow cytometry measuring Ki-67 nuclear antigen expression in MCF-7 cells treated for $24 \mathrm{~h}$ with TAM $(1 \mu \mathrm{M})$ in combination with (i) IVIG $(50 \mu \mathrm{g} / \mathrm{mL})$; (ii) anti-ER $\alpha$ Abs $(50 \mu \mathrm{g} / \mathrm{mL})$; (iii) IVIG $(50 \mu \mathrm{g} / \mathrm{mL})$ plus simvastatin $(2.5 \mu \mathrm{M})$; (iv) anti-ER $\alpha$ Abs $(50 \mu \mathrm{g} / \mathrm{mL})$ plus simvastatin $(2.5 \mu \mathrm{M})$. Data are reported as mean $\pm \mathrm{SD}$ of five independent experiments. TAM plus anti-ER $\alpha$ Abs vs. each other condition, ${ }^{*}, p<0.05$ by Student's $t$ test. (b) Cell-cycle phases were determined by flow cytometry analysis of BrdU/7AAD staining in synchronized MCF-7 cells treated as reported in panel a. Data are reported as mean \pm SD of each phase of five independent experiments. TAM plus anti-ER $\alpha$ Abs vs. each other condition for G0/G1 phase ${ }^{* *}, p<0.01$ by Student's $t$ test. (c) The mean $( \pm \mathrm{SD}$ ) of the tumor volume of one set of experiment (five mice/group) was shown. The tumor volumes were calculated using the following formula: $(a \times b 2) / 2$, where $a$ and $b$ represent the longest and shortest diameters, respectively. At day 14, TAM plus anti-ER $\alpha$ Abs vs. each other condition, ${ }^{* * *}, p<0.001$ by Student's $t$ test. (d) Representative sections from MCF-7 tumors treated with TAM + IVIG + Simva and TAM + anti-ER $\alpha$ Abs + Simva, immunostained with anti-Ki67 (red) antibodies and counterstained with Hoechst (blue) are shown. Ki-67 was scored by the average method as reported in Methods. Results are also represented as mean \pm SD. Scale bar $=50 \mu \mathrm{m}$. (e) Western blot analysis of cyclin $\mathrm{E}$ in tumor lysates. For each cell line, blots shown are representative of five independent experiments (left). Densitometry analysis of specific protein levels relative to Hsc70 is also shown (right). Values are expressed as mean \pm SD; TAM plus anti-ER $\alpha$ Abs vs. each other condition, **, $p<0.01$ by Student's $t$ test. Simva: simvastatin.

Summarizing, reducing cholesterol-rich lipid raft domains by statins could restore TAM sensitivity in breast cancer, preventing the anti-ER $\alpha$ Abs-mediated TAM resistance (Figure 5). Interestingly, several clinical trials are ongoing to elucidate the capability of statins to prevent chemotherapy-mediated cardiovascular toxicity, or to lower cholesterol increase related to aromatase inhibitors administration, but also to interfere with breast cancer growth. This study provides support for the further evaluation of simvastatin in combination to TAM as a new strategy for overcoming endocrine resistance in ER-positive breast cancer patients positive for serum anti-ER $\alpha$ Abs. 


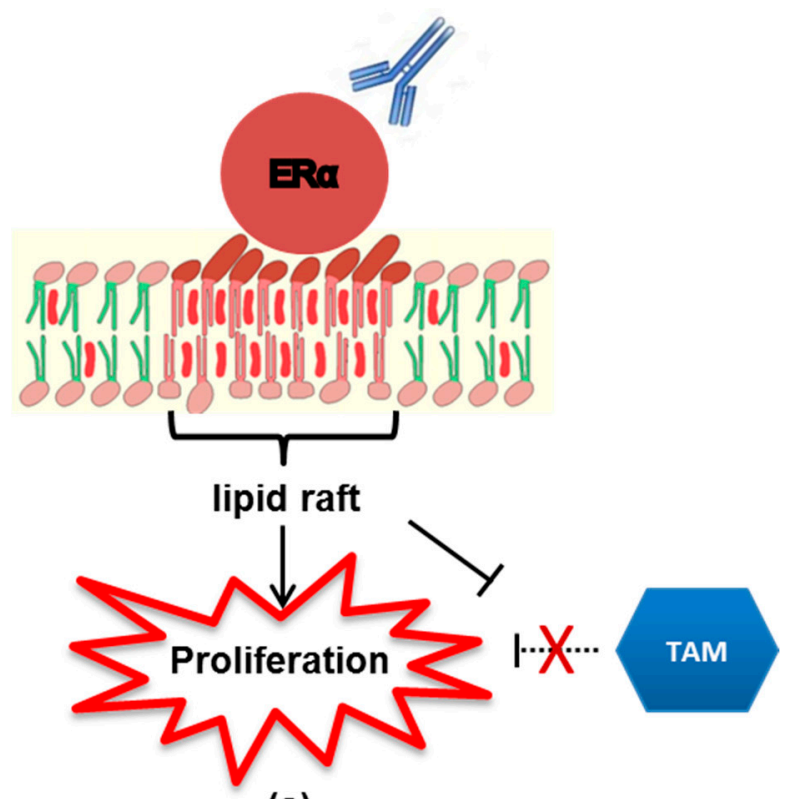

(a)

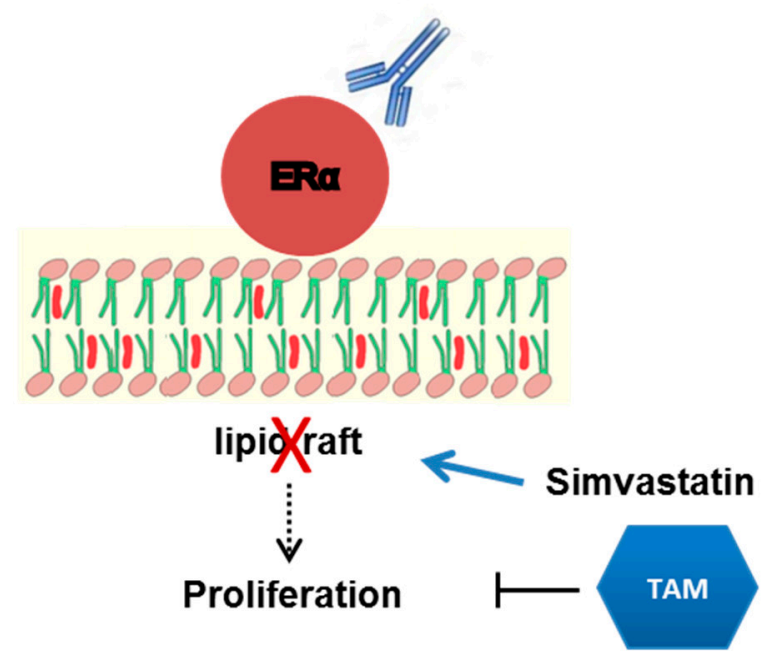

(b)

Figure 5. A model showing the effect of anti-ER $\alpha$ Abs on MCF-7 cells. (a) Anti-ER $\alpha$ Abs bind to and activate membrane ERalpha inducing MCF-7 cell proliferation. The "anti-ER $\alpha$ Abs-membrane ER" mediated signaling acts as a negative-regulatory factor of the tamoxifen-induced inhibition of cell growth. (b) Since membrane ERalpha is embedded in lipid rafts, the disaggregation of these rafts by simvastatin hampers the "anti-ER $\alpha$ Abs-membrane ER" mediated signaling, blocks anti-ER $\alpha$ Abs-mediated cell proliferation and restores tamoxifen inhibition.

In conclusion, at the best of our knowledge, this is the first study in which a simple and non-invasive analysis, i.e., the search of autoantibodies in sera of patients, is shown to provide useful data concerning the risk of occurrence of TAM resistance and its prevention in ER-positive breast cancer patients. The provided clues, if confirmed in further studies, may significantly integrate decision concerning TAM administration at an individual patient level, and more generally lead the way to new research pipelines clarifying the underlying mechanisms of resistance in ER-positive breast cancer patients candidates to endocrine therapy. 
Supplementary Materials: The following are available online at http://www.mdpi.com/2073-4409/8/7/750/s1, Figure S1: SDS-PAGE under reducing condition analysis (Coomassie brilliant blue staining) of human anti-ER $\alpha$ Abs obtained from early ER-positive breast cancer patients., Figure S2: Cell proliferation evaluated by flow cytometry measuring Ki-67 nuclear antigen expression in MCF-7 cells treated or not for $24 \mathrm{~h}$ with E2 (10 nM), E2 + anti-ER $\alpha$ Abs $(50 \mu \mathrm{g} / \mathrm{mL}), \mathrm{E} 2+\mathrm{TAM} 1 \mu \mathrm{M}, \mathrm{E} 2+$ anti-ER $\alpha$ Abs + TAM.

Author Contributions: Conceptualization, A.M., M.B., M.P., E.O.; investigation, A.M., S.P., R.P., C.R., M.P., M.S., D.M., G.P., E.I., M.T.P.; methodology, F.C., L.G., A.C., P.M.; resources, P.V., M.B., L.P.; project administration E.O.; supervision and validation, A.C., P.V., P.M., M.P., E.O.; writing, S.P., R.P., M.T.P., P.M., M.P., E.O.

Funding: This work was supported by AIRC (IG16810 to EO) and partially supported by AIRC (18526 to PM) and Peretti Foundation (PM).

Acknowledgments: This paper may be considered as a tribute to our young investigator Angela Maselli who passed away prematurely during the writing of this manuscript. Her enthusiastic and qualified contribution was essential for the achievement of this study. We are grateful to Chiara Mandoj and Giacomo Barchiesi for their technical support in sample collection and classification.

Conflicts of Interest: The authors disclose no potential conflicts of interest.

\section{References}

1. Davies, C.; Godwin, J.; Gray, R.; Clarke, M.; Cutter, D.; Darby, S.; McGale, P.; Pan, H.C.; Taylor, C.; Wang, Y.C.; et al. Relevance of breast cancer hormone receptors and other factors to the efficacy of adjuvant tamoxifen: Patient-level meta-analysis of randomised trials. Lancet 2011, 378, 771-784. [CrossRef] [PubMed]

2. EBCTCTG. Effects of chemotherapy and hormonal therapy for early breast cancer on recurrence and 15-year survival: An overview of the randomised trials. Lancet 2005, 365, 1687-1717. [CrossRef]

3. Jemal, A.; Siegel, R.; Ward, E.; Hao, Y.; Xu, J.; Thun, M.J. Cancer statistics, 2009. CA Cancer J. Clin. 2009, 59, 225-249. [CrossRef] [PubMed]

4. Arnal, J.F.; Lenfant, F.; Metivier, R.; Flouriot, G.; Henrion, D.; Adlanmerini, M.; Fontaine, C.; Gourdy, P.; Chambon, P.; Katzenellenbogen, B.; et al. Membrane and Nuclear Estrogen Receptor Alpha Actions: From Tissue Specificity to Medical Implications. Physiol. Rev. 2017, 97, 1045-1087. [CrossRef]

5. Levin, E.R.; Hammes, S.R. Nuclear receptors outside the nucleus: Extranuclear signalling by steroid receptors. Nat. Rev. Mol. Cell Biol. 2016, 17, 783-797. [CrossRef]

6. Marino, M.; Ascenzi, P. Membrane association of estrogen receptor alpha and beta influences 17beta-estradiol-mediated cancer cell proliferation. Steroids 2008, 73, 853-858. [CrossRef] [PubMed]

7. Shiau, A.K.; Barstad, D.; Loria, P.M.; Cheng, L.; Kushner, P.J.; Agard, D.A.; Greene, G.L. The structural basis of estrogen receptor/coactivator recognition and the antagonism of this interaction by tamoxifen. Cell 1998, 95, 927-937. [CrossRef]

8. Ali, S.; Coombes, R.C. Endocrine-responsive breast cancer and strategies for combating resistance. Nat. Rev. Cancer 2002, 2, 101-112. [CrossRef]

9. Clarke, R.; Tyson, J.J.; Dixon, J.M. Endocrine resistance in breast cancer-An overview and update. Mol. Cell Endocrinol. 2015, 418, 220-234. [CrossRef]

10. Musgrove, E.A.; Sutherland, R.L. Biological determinants of endocrine resistance in breast cancer. Nat. Rev. Cancer 2009, 9, 631-643. [CrossRef]

11. Gu, W.; Dong, N.; Wang, P.; Shi, C.; Yang, J.; Wang, J. Tamoxifen resistance and metastasis of human breast cancer cells were mediated by the membrane-associated estrogen receptor ER-alpha36 signaling in vitro. Cell Biol. Toxicol. 2017, 33, 183-195. [CrossRef] [PubMed]

12. Wang, Z.Y.; Yin, L. Estrogen receptor alpha-36 (ER-alpha36): A new player in human breast cancer. Mol. Cell Endocrinol. 2015, 418, 193-206. [CrossRef] [PubMed]

13. Maselli, A.; Capoccia, S.; Pugliese, P.; Raggi, C.; Cirulli, F.; Fabi, A.; Malorni, W.; Pierdominici, M.; Ortona, E. Autoantibodies specific to estrogen receptor alpha act as estrogen agonists and their levels correlate with breast cancer cell proliferation. Oncoimmunology 2015, 5. [CrossRef] [PubMed]

14. Pierdominici, M.; Maselli, A.; Locatelli, S.L.; Ciarlo, L.; Careddu, G.; Patrizio, M.; Ascione, W.; Matarrese, P.; Oetona, E. Estrogen receptor beta ligation inhibits Hodgkin lymphoma growth by inducing autophagy. Oncotarget 2017, 8, 8522-8535. [CrossRef] [PubMed] 
15. Riemann, D.; Tcherkes, A.; Hansen, G.H.; Wulfaenger, J.; Blosz, T.; Danielsen, E.M. Functional co-localization of monocytic aminopeptidase N/CD13 with the Fc gamma receptors CD32 and CD64. Biochem. Biophys. Res. Commun. 2005, 331, 1408-1412. [CrossRef] [PubMed]

16. Pajak, B.; Wojewodzka, U.; Gajkowska, B.; Orzechowski, A. Lipid rafts in anticancer therapy: Theory and practice (Review). Mol. Med. Rep. 2008, 1, 167-172. [PubMed]

17. Simons, K.; Toomre, D. Lipid rafts and signal transduction. Nat. Rev. Mol. Cell Biol. 2000, 1, 31-39. [CrossRef] [PubMed]

18. Marquez, D.C.; Chen, H.W.; Curran, E.M.; Welshons, W.V.; Pietras, R.J. Estrogen receptors in membrane lipid rafts and signal transduction in breast cancer. Mol. Cell Endocrinol. 2006, 246, 91-100. [CrossRef]

19. Osborne, C.K.; Bardou, V.; Hopp, T.A.; Chamness, G.C.; Hilsenbeck, S.G.; Fuqua, S.A.W.; Wang, J.; Allred, D.C.; Clark, G.M.; Schiff, R. Role of the estrogen receptor coactivator AIB1 (SRC-3) and HER-2/neu in tamoxifen resistance in breast cancer. J. Natl. Cancer Inst. 2003, 95, 353-361. [CrossRef]

20. Pedram, A.; Razandi, M.; Aitkenhead, M.; Hughes, C.C.; Levin, E.R. Integration of the non-genomic and genomic actions of estrogen. Membrane-initiated signaling by steroid to transcription and cell biology. J. Biol. Chem. 2002, 277, 50768-50775. [CrossRef]

21. Mahammad, S.; Parmryd, I. Cholesterol depletion using methyl-beta-cyclodextrin. Methods Mol. Biol. 2015, 1232, 91-102. [CrossRef] [PubMed]

22. Beckwitt, C.H.; Brufsky, A.; Oltvai, Z.N.; Wells, A. Statin drugs to reduce breast cancer recurrence and mortality. Breast Cancer Res. 2018, 20, 144. [CrossRef] [PubMed]

23. Inoue, K.; Fry, E.A. Novel Molecular Markers for Breast Cancer. Biomark. Cancer 2016, 8, 25-42. [CrossRef] [PubMed]

24. Sutherland, R.L.; Musgrove, E.A. Cyclins and breast cancer. J. Mammary Gland Biol. Neoplasia 2004, 9, 95-104. [CrossRef] [PubMed]

(C) 2019 by the authors. Licensee MDPI, Basel, Switzerland. This article is an open access article distributed under the terms and conditions of the Creative Commons Attribution (CC BY) license (http://creativecommons.org/licenses/by/4.0/). 